Research letter

\title{
Complex biomechanical study of chitosan microtubes
}

\author{
Natalia O. Gegel, Anastasia A. Golyadkina, Tatyana S. Babicheva, Xenia K. Skripachenko, Victoria Yu. Papkina
}

Saratov State University, Saratov, Russia

Received 21 October 2017, Revised 13 September 2018, Accepted 26 September 2018

(c) 2017, Gegel N.O., Golyadkina A.A., Babicheva T.S., Skripachenko X.K., Papkina V.Yu.

(C) 2017, Russian Open Medical Journal

Abstract: Mechanical parameters of a biotransplant made of the aminopolysaccharide chitosan were estimated in an in vitro experiment. Using the values obtained, finite element simulation of the "arterial vessel-microtube" system was carried out. As a result of our numerical experiment, the distributions of hemodynamic values were calculated taking into account the stress-strain state of the arterial wall adjacent to the transplant. The results obtained allow predicting the behavior of the material under study in the human body under various loads.

Keywords: chitosan, microtubes, blood vessel prosthesis, in vitro experiment, finite element simulation.

Cite as Gegel NO, Golyadkina AA, Babicheva TS, Skripachenko XK, Papkina VYu. Complex biomechanical study of chitosan microtubes. Russian Open Medical Journal 2019; 8: e0103.

Correspondence to Natalia Gegel. Address: 83, Astrakhanskaya str., Saratov, 410012, Russia. Tel.: +79626230662. E-mail: gegelno@yandex.ru.

\section{Introduction}

At present, approaches to the production of alternative transplants for vascular tissue regeneration are widely developed in bioengineering [1]. This is largely due to cardiovascular diseases, which are the most common in the world, especially in pediatric surgery, because modern synthetic prosthesis is incapable of adapting to an ever-growing, evolving organism [2]. The literature contains a large number of publications reflecting attempts to make transplants from synthetic polymers and biopolymers [3-5]. Nevertheless, not all existing models prostheses are close enough (for biomechanical properties) to the native arteries, leading to numerous operational complications caused by biomechanical incompatibility $[6,7]$. The indicators traditionally recorded (the tensile stress and relative deformation of a product) do not adequately reflect the elastic-deformation properties of vascular prostheses when they are introduced into the living body. Finite element simulation is an informative method for predicting the possible behavior of transplants in the living organism $[8,9]$. Earlier we developed a method for obtaining hollow cylindrical chitosan microtubes of various diameters [10]. Our evaluation of their morphology, elastic-plastic and biochemical parameters has allowed considering our chitosan microtubes as a promising model of vascular transplants $[11,12]$.

The purpose of this study was to examine the physicomechanical properties of chitosan microtube samples under conditions as close to native as possible and to perform finite element simulation using the parameters obtained.

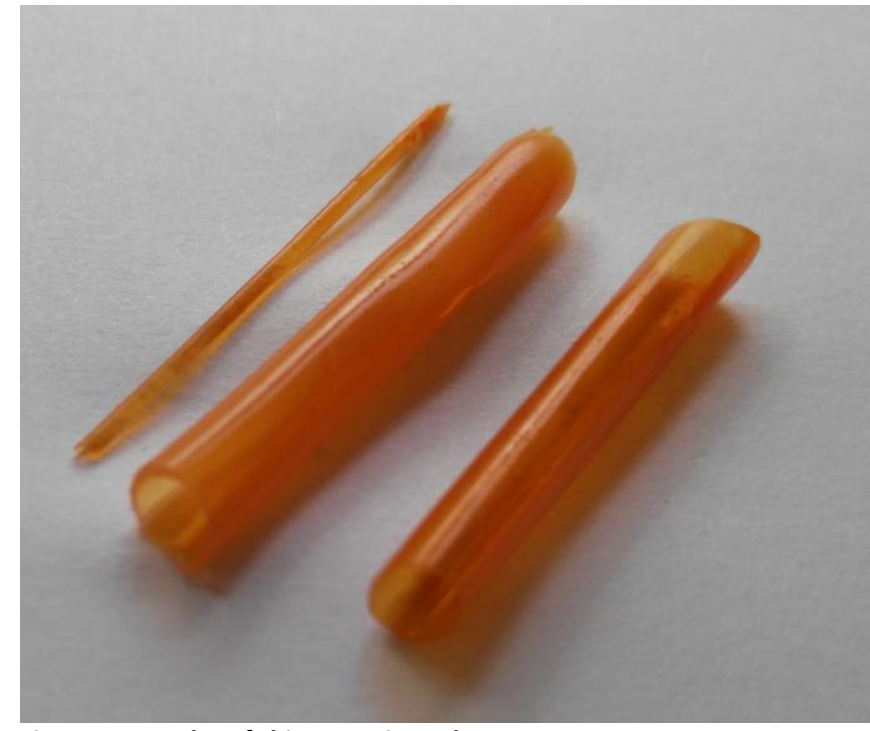

Figure 1. Samples of chitosan microtubes

\section{Material and Methods}

Microtubes with an internal diameter of $5 \mathrm{~mm}$ obtained by a dry molding method from 4 wt.\% chitosan solution in $1.5 \%$ glycolic acid were selected as the objects of our study (Figure 1). These methods are underlain by the processes of phase separation and the polysalt $\rightarrow$ polybase conversion reaction Microtubes were formed as follows [10]. We used a glass rod with a circular cross section of $5 \mathrm{~mm}$ in diameter, immersed it vertically in a solution of 
chitosan and held for 1 minute to evenly cover the surface layer of the solution forming. The chitosan-coated rod was then immersed in an aqueous solution of the salting-out agent for $1 \mathrm{~min}$ to form a first layer of the water-insoluble polymer, removed, and dried in an oven at $45-50^{\circ} \mathrm{C}$ for $4-5 \mathrm{~h}$ until complete evaporation of the solvent. A condensed phase of the polymer was thus formed. Then, the rod was re-immersed into the aqueous solution of the salting-out agent to form a second layer of the water-insoluble polymer and, accordingly, the microtube walls as a hollow cylinder. These microtube samples were removed from the rod, washed with distilled water until neutral, and kept in a swollen state in distilled water.

$\mathrm{NaOH}-\mathrm{NaOH}$ (Sample 1) and triethanolamine (TEA)- $\mathrm{NaOH}$ (Sample 2) were used as reagents for the polysalt $\rightarrow$ polybase transformation to form the walls of these microtubes. To improve the mechanical properties, a modifier (polyethylene oxide) had been introduced into the molding solution which sample 1 was prepared from (Sample 3). Addition of polyethylene oxide additives into a chitosan solution is known to enhance the intermolecular interactions, resulting in the formation of a stronger fluctuation network of crosslinks [13].

Physico-mechanical tests were carried out on an Instron 5944 tensile machine. The uniaxial tensile test provides data that can be used to determine the mechanical properties of the material, such as the modulus of elasticity (Young's modulus), tensile stress at tension, and relative deformation. The modulus of elasticity characterizes the material's resistance to stretching during deformation. In other words, the greater the modulus of elasticity, the less the sample is deformed. The breaking voltage characterizes the value of the voltage above which the material is destroyed. The tensile test is carried out within the maximum force of the load frame. In our study we used a sensor with an allowable load of $50 \mathrm{~N}$. The determination of the mechanical properties of the material proceeded as follows: the opposite ends of the sample are placed between the clamps of the testing machine; the geometry of the sample is determined and dimensions are taken (length, width, thickness or radius); the traverse speed is set. After starting the test, the traverse with the upper clamp moves at the specified speed, stretching the sample. With deformation, resistance (force) appears in it. The test ends when the material is destroyed. During stretching, information about the elongation of the specimen, the speed of the traverse and the resistance tested by the specimen are monitored and recorded. The loading rate was $10 \mathrm{~mm} / \mathrm{min}$. It is known that arteries and, accordingly, vascular prosthesis are in a moistened state in the human body. Due to this, mechanical properties were studied using a liquid-medium test chamber (BioBath) at room temperature in a $0.9 \%$ aqueous solution of sodium chloride (saline), which increased the accuracy of the results. All mechanical tests were carried out while maintaining the original shape of the microtube as a hollow cylinder. Experiments were conducted under cyclic loading-unloading to evaluate the stability of our samples. The number of cycles was 500 with a $10 \%$ deformation. After cyclic loading, the main stretching of the sample began, where the deformations reached values of more than $10 \%$. The end of the test was the load drop of $40 \%$. As a result of full-scale experiments, the values of Young's modulus (modulus of elasticity), rupture voltage and relative strain. The obtained mechanical parameters were used for finite element simulation (FES).
Table 1. Average values of the mechanical parameters for chitosan samples and arterial wall

\begin{tabular}{lccc}
\hline Material type & Young's modulus, $\mathrm{MPa}$ & Br.Str., $\mathrm{MPa}$ & $\mathrm{MRE}$ \\
\hline Sample 1 & 5.11 & 0.9 & 0.22 \\
Sample 2 & 8.88 & 1.47 & 0.22 \\
Sample 3 & 11.45 & 1.29 & 0.13 \\
Arterial wall (the inner & 5.44 & 0.40 & 0.80 \\
carotid artery) & & &
\end{tabular}

Br.Str., breaking stress; MRE, Maximum relative elongation.

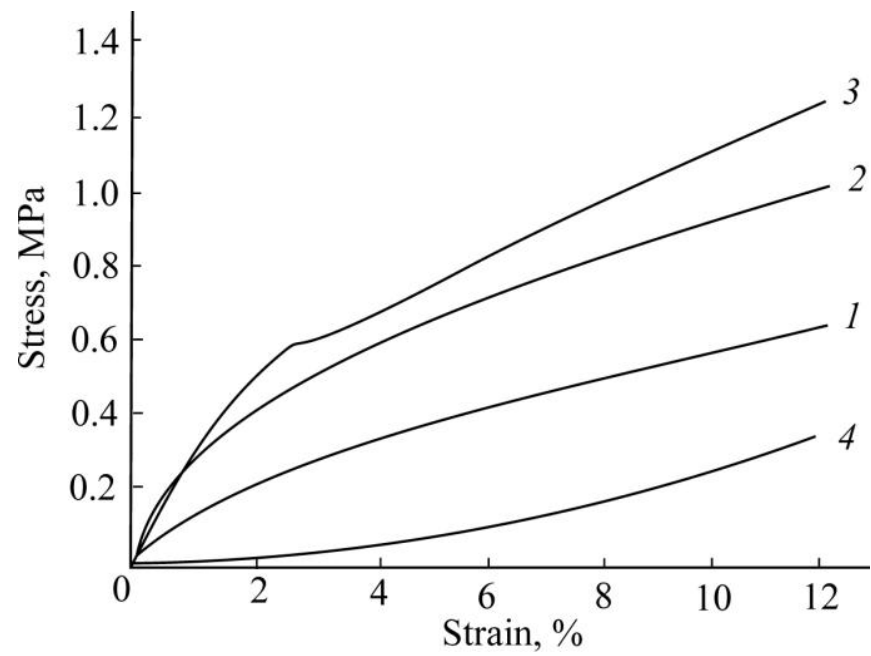

Figure 2. Averaged stress-strain curves for microtube samples: 1 (1), 2 (2), 3 (3), and the arterial wall (4).

To predict the behavior of a microtube in the human body, finite element simulation of the "arterial vessel-microtube" system was carried out. A model was built in the SolidWorks CAD system. A numerical experiment was implemented in the AnsysWorkBench software using the finite element method. A model of an arterial vessel prosthetized with a chitosan microtube was built. The material of the arterial wall was assumed to be hyperelastic and isotropic with the mechanical parameters obtained earlier in the course of in vitro experiments [14]. The microtube material was considered to be perfectly elastic and isotropic with the parameters obtained in the course of in vitro experiments. The blood was considered as a homogeneous, incompressible and Newtonian fluid with a dynamic viscosity of $\mu=1.050 \mathrm{~m} / \mathrm{s}$ and a density of $\rho=0.0037 \mathrm{~kg} / \mathrm{m}^{3}$. The ends of the vessel were rigidly fixed. The condition for the equality of the velocities of the fluid particles adjacent to the corresponding wall elements was set on the walls of the artery and microtube. Zero pressure was applied to the artery outlet. At the inlet, the time velocity function was set, varying according to the physiological law. As a result of our numerical experiment, the distributions of hemodynamic values were obtained, taking into account the stress-strain state of the arterial wall adjacent to the transplant.

\section{Results}

As a result of our in vitro experiments, the mechanical parameters of the microtubes were estimated, namely: Young's modulus, the breaking stress and elongation (Table 1). The curves of the "relative elongation-stress" dependence were plotted (Figure 2). The mechanical parameters of the arterial wall are given in the table and on the plot for comparative analysis. 


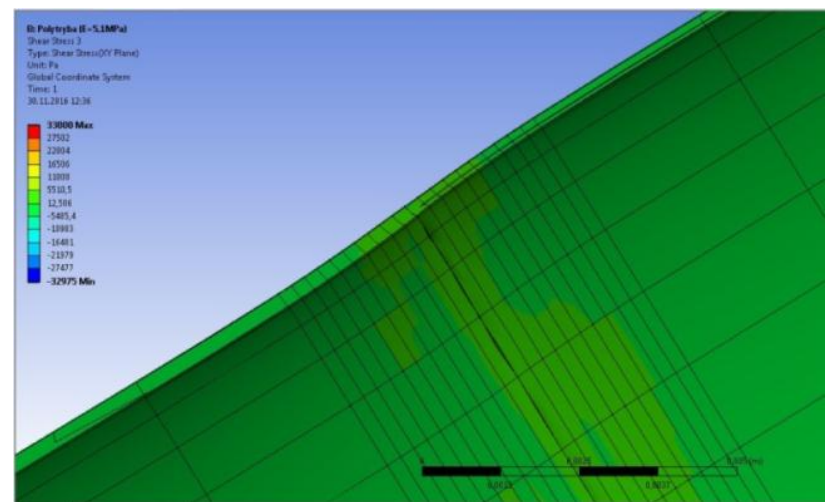

Figure 3. Effective von Mises stresses for the "arterial vessel - Sample 1» model.

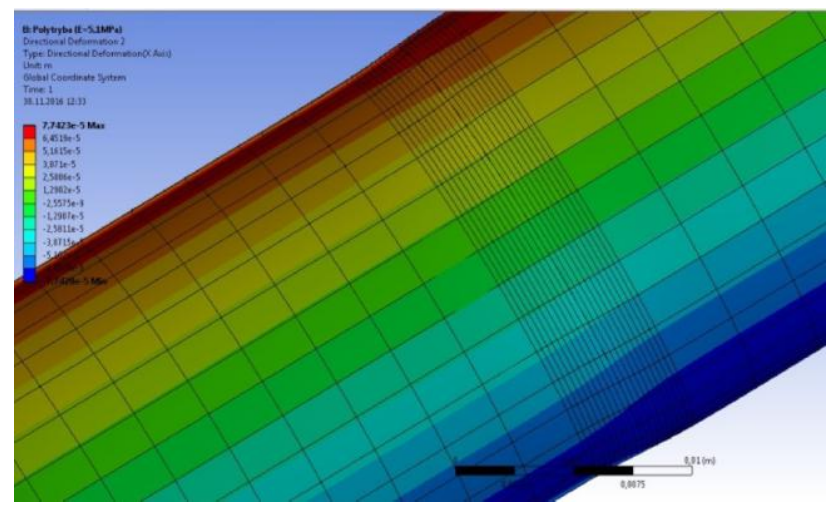

Figure 4. Deformations for the "arterial vessel - microtube Sample 1» model.
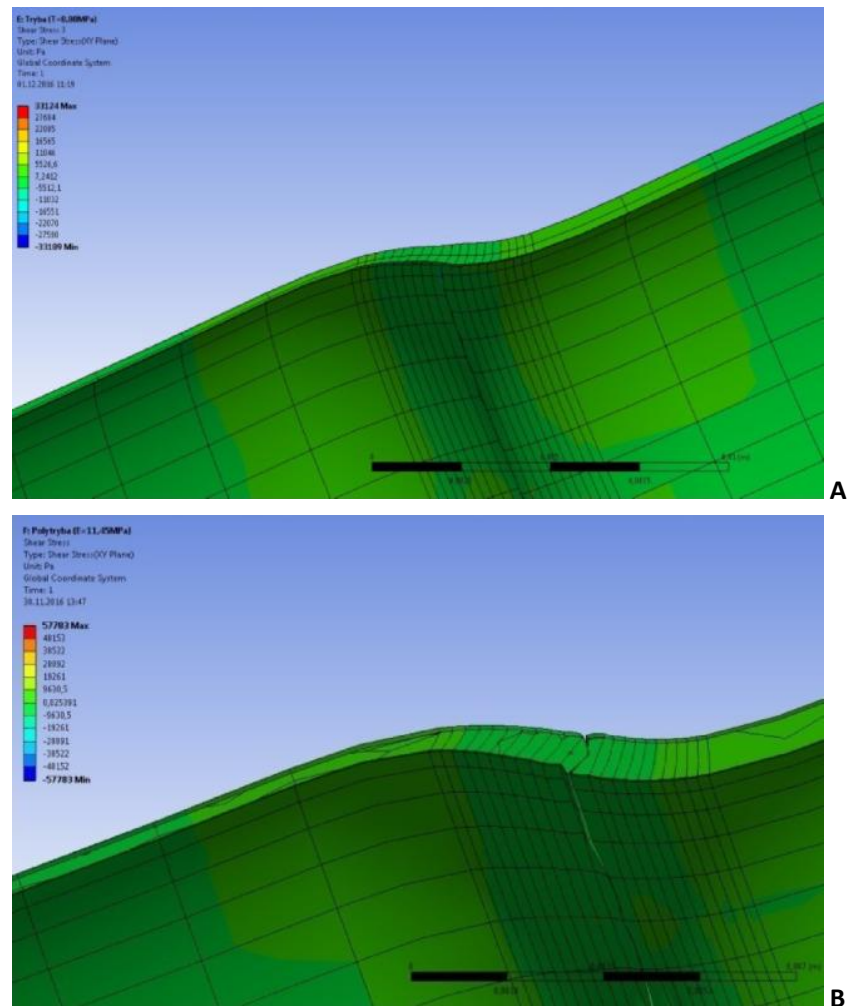

Figure 5. Effective von Mises stresses for the «arterial vessel - Sample 2» (A) and «arterial vessel - Sample 3» models (B).
Analysis of our experimental data has shown that Sample 3 possesses a high Young modulus, rather than other tissues. The Young modulus of Sample 1 is very close to that of the arterial wall. It was revealed that none of the chitosan samples reached the limiting value of relative elongation of the arterial wall. It has been found that the relative elongation and breaking stress for Sample 1 and the arterial wall are closest in comparison with the other samples studied.

In the course of our numerical experiment, problems with the Young moduli of all transplant samples were solved in accordance with the results of in vitro experiments. As a result of our numerical experiment, the distributions of hemodynamic values were obtained, taking into account the stress-strain state of the arterial wall adjacent to the transplant. The results of the stress and deformation distribution at the area of contact between the arterial wall and the transplant are interesting from a practical viewpoint.

The results of the numerical experiment for the "arterial vessel - Sample 1" model show a uniform distribution of the equivalent stresses (Figure 3) and displacements (Figure 4). This is due to the close values of the Young moduli of the materials under study.

When estimating equivalent stresses, the zone of contact with the arterial wall was unstable in Samples 2 and 3 (Figure 5). This is due to the fact that the Young modulus of the arterial wall is inferior in value to that of the Samples used (Table 1).

\section{Discussion}

Analysis of the results of our numerical experiments has made it possible to reveal instability zones localized in the area of contact between the vessel's walls and the microtube. This factor, possibly under certain conditions, could lead to destruction of the transplant, which was observed when considering the "arterial vessel - Sample 2" and "arterial vessel - Sample 3" models. This is perhaps due to some peculiarities of the chemical reaction during microtube formation. Gegel et al. [10] evaluated the influence of the salting-out agent nature on the supramolecular structure of the polymeric system formed. The use of an organic or inorganic base as the salting-out agent leads to the formation of a microtube wall with a relatively dense porous supramolecular structure formed similarly to a physical crosslink network and stabilized by hydrogen bonding. PEO addition promotes the formation of a more developed porous surface of the microtube wall. This character of the inner structure apparently affects the elasticmechanical properties of the microtubes as well.

When evaluating our in vitro experiments, it is of interest that Sample 1 is very close (by mechanical parameters) to the arterial wall. However, it reaches no more than $25 \%$ of the limiting value of relative elongation of the arterial wall (Table 1). Further work will be directed to searching for modifiers capable of attaching our microtubes higher tensile values. It should be noted that our approach to obtaining chitosan microtubes is innovative. In the world scientific literature, only one work has been found in which a tubular frame with high mechanical strength, bio- and hemocompatibility, comparable to the main characteristics of tissue-engineering vascular graft, was obtained from a mixture of synthetic polymer with gelatin by electroforming method [15]. 


\section{Conclusion}

Thus, the conducted in vitro and numerical experiments of microtubule samples showed that the sample of a microtubule obtained using an organic base is nearly as close to native tissue as possible compared to other samples. For the first time, finite element modeling of the system "arterial vessel - microtube of chitosan" was carried out. The uniform distribution of values of equivalent stresses and displacements in the area of contact between the arterial wall and the graft is revealed. In the course of complex studies, the comparatively simple method of obtaining seamless hollow cylindrical structures (microtubes) from chitosan of different diameters in solution technology, developed by us, allows us to consider them as a promising model of analogues of vascular grafts.

\section{Ethical approval}

This article does not contain any studies with human participants or animals performed by any of the authors.

\section{Funding}

This work was supported by the Russian Foundation for Basic Research, project No 17-33-00953 «Scientific fundamentals of the preparation of 3D biodegradable structures from natural polysaccharides to create blood vessel analogs".

\section{Conflict of Interests}

The authors declare that there is no conflict of interests regarding the publication of this paper.

\section{Reference}

1. Benrashid E, McCoy CC, Youngwirth LM, Kim J, Manson RJ, Otto JC, Lawson JH. Tissue engineered vascular grafts: origins, development, and current strategies for clinical application. Methods 2016; 99: 1319. https://doi.org/10.1016/i.ymeth.2015.07.014.

2. Heydrick S, Roberts E, Kim J, Emani S, Wong JY. Pediatric cardiovascular grafts: historical perspective and future directions. Curr Opin Biotechnol 2016; 40: 119-124. https://doi.org/10.1016/i.copbio.2016.03.013.

3. Melchiorri AJ, Hibino N, Best CA, Yi T, Lee YU, Kraynak CA, et. al. 3Dprinted biodegradable polymeric vascular grafts. Adv Healthc Mater 2016; 5(3): 319-325. https://doi.org/10.1002/adhm.201500725.

4. Marcolin C, Draghi L, Tanzi M, Faré S. Electrospun silk fibroin-gelatin composite tubular matrices as scaffolds for small diameter blood vessel regeneration. J Mater Sci Mater Med Med 2017; 28: 80-86. https://doi.org/10.1007/s10856-017-5884-9.

5. Chongab DST, Constantinoua J, Davisa M, Hamiltonab G. Calcification of a synthetic renovascular graft in a child. EJVES Short Rep 2016; 33: 13-15. https://doi.org/10.1016/j.ejvssr.2016.06.001.

6. Spadaccio C, Nappi F, Al-Attar N, Sutherland FW, Acar C, Nenna A, et al. Old myths, new concerns: the long-term effects of ascending aorta replacement with dacron grafts. Not all that glitters is gold. J Cardiovasc Transl Res 2016; 9(4): 334-342. https://doi.org/10.1007/s12265-016-9699-8.

7. Askari F, Shafieian M, Solouk A, Hashemi A. A comparison of the material properties of natural and synthetic vascular walls. J Mech Behavior Biomed Mater 2017; 71: 209-215. https://doi.org/org/10.1016/j.jmbbm.2017.03.016.

8. Kossovich LY, Kirillova IV, Golyadkina AA, Polienko AV, Chelnokova NO, Ivanov DV, et al. Patient-specific modeling of human cardiovascular system elements. Proc SPIE 2016; 9710: 971014. https://doi.org/10.1117/12.2208426.

9. Popova IV, Stepanova AO, Sergeevichev DS, Akulov AE, Zakharova IS, Pokushalov $A A$, et al. Comparative study of three vascular grafts produced by electrospinning in vitro and in vivo. Circulation Pathology and Cardiac Surgery 2015; 19(4): 63-71. Russian. https://elibrary.ru/item.asp?id=25035638.

10. Gegel NO, Babicheva TS, Shipovskaya AB. Peculiarities obtaining of microtubes chitosan interfacial reaction of polymer-analogous transformations. Butlerov Communications 2015; 41(3): 44-53. Russian. https://elibrary.ru/item.asp?id=23561990.

11. Babicheva TS, Gegel NO, Shipovskaya AB. Influence of the salting-out agent nature on the strength properties of chitosan microtubes. J Nat Sci Sustainable Techn 2015; 9(2): 2-17. http://www.novapublishers.org/catalog/product_info.php?products i $\mathrm{d}=54491$.

12. Gegel NO, Babicheva TS, Shipovskaya AB. Wall morphology of chitosan-based hollow cylindrical materials with a layered structure. BioNanoScience 2018; 8(2): 661-667. https://doi.org/10.1007/s12668 017-0415-1.

13. Kolsanova EV, Shmakov SL, Shipovskaya AB. System chitosan-acetic acid-water with the addition of polyethylene oxide and nonwoven materials on its basis. LAP Lambert Academic Publishing, 2014; 236 p.

14. Golyadkina AA, Ivanov DV, Kirillova IV, Kossovich EL, Pavlova OE, Polyenko AV, Safonov RA. Biomechanics of the carotid artery. Saratov, Russia: Izdatel'stvo "Saratovskiy istochnik", 2015; 174 p. Russian. https://elibrary.ru/item.asp?id=25334342.

15. Vatankhah E, Prabhakaran MP, Semnani D, Razavi S, Morshed M Ramakrishna S. Electrospun tecophilic/gelatin nanofibers with potential for small diameter blood vessel tissue engineering. Biopolymers 2014; 101(12): 1165-1180. https://doi.org/10.1002/bip.22524

Authors:

Natalia O. Gegel - PhD, Deputy Head of Department, Educational-Research Institute of Nanostructures and Biosystems, Saratov State University, Saratov, Russia. http://orcid.org/0000-0001-5724-7571.

Anastasiya A. Golyadkina - PhD, Head Researcher, Educational-Research Institute of Nanostructures and Biosystems, Saratov State University, Saratov, Russia. http://orcid.org/0000-0003-0587-8691.

Tatyana S. Babicheva - Head Assistant, Institute of Chemistry, Saratov State University, Saratov, Russia. http://orcid.org/0000-0002-6655-8483.

Xenia K. Skripachenko - Engineer, Educational-Research Institute of Nanostructures and Biosystems, Saratov State University, Saratov, Russia. http://orcid.org/0000-0001-6156-1854.

Victoria Yu. Papkina - Student, Institute of Chemistry, Saratov State University, Saratov, Russia. https://orcid.org/0000-0002-4520-1733. 